\title{
Association of cortactin with dynamic actin in lamellipodia and on endosomal
} vesicles

\author{
Marko Kaksonen ${ }^{1, *}$, H. Benjamin Peng ${ }^{2}$ and Heikki Rauvala ${ }^{1}$ \\ ${ }^{1}$ Laboratory of Molecular Neurobiology, Department of Biosciences and Institute of Biotechnology, PO Box 56, FIN-00014, \\ University of Helsinki, Finland \\ 2Department of Cell Biology and Anatomy, Neuroscience Center and Curriculum in Neurobiology, University of North Carolina at \\ Chapel Hill, Chapel Hill, North Carolina, 27599, USA \\ *Author for correspondence (e-mail: marko.kaksonen@helsinki.fi) \\ Accepted 4 October; published on WWW 16 November 2000
}

\section{SUMMARY}

We have used fluorescent protein tagging to study the localization and dynamics of the actin-binding protein cortactin in living NIH 3 T3 fibroblast cells. Cortactin was localized to active lamellipodia and to small cytoplasmic spots. Time-lapse imaging revealed that these cortactin labeled structures were very dynamic. In the lamellipodia, cortactin labeled structures formed at the leading edge and then moved toward the cell center. Experiments with green fluorescent protein (GFP)-tagged actin showed that cortactin movement was coincident with the actin retrograde flow in the lamellipodia. Cytoplasmic cortactin spots also contained F-actin and were propelled by actin polymerization. Arp3, a component of the arp2/3 complex which is a key regulator of actin polymerization, colocalized with cortactin. Cytoplasmic cortactin-labeled spots were found to be associated with endosomal vesicles. Association was asymmetric and approximately half of the endosomes were associated with cortactin spots. Time-lapse imaging suggested that these cortactin and F-actin-containing spots propelled endosomes. Actin polymerization based propulsion may be a common mechanism for endomembrane trafficking in the same manner as used in the plasma membrane protrusions. As cortactin is known to interact with membrane-associated signaling proteins it could have a role in linking signaling complexes with dynamic actin on endosomes and in lamellipodia.

\section{Movies available on-line: \\ http://www.biologists.com/JCS/movies/jcs1893.html}

Key words: Cortactin, Actin assembly, Arp 2/3 complex, Endocytosis, Cell motility

\section{INTRODUCTION}

The formation of cell protrusions such as lamellipodia and filopodia in motile cells is dependent on actin polymerization. The actin assembly takes place at the leading edge of the protrusions and is thought to provide the force for forward movement of the plasma membrane (for review see Mitchison and Cramer, 1996). In several cell types the actin meshwork moves in a retrograde manner from the leading edge toward the cell body where actin is then depolymerized (Fisher et al., 1988; Theriot and Mitchison, 1992; Wang, 1985). This movement is known to be myosin dependent (Lin et al., 1996). It has been suggested that attachment of the actin cytoskeleton in lamellipodia to the substrate via transmembrane adhesion receptors stops the retrograde movement and leads to forward movement of the cell body (Lin and Forscher, 1995; Suter et al., 1998). This is supported by the finding that in some very rapidly migrating cell types the actin meshwork in the lamellipodia stays immobile in relation to the substrate (Theriot and Mitchison, 1991).

Actin cytoskeleton has also been shown to be crucial for transport of endocytosed molecules. The initial internalization step requires actin cytoskeleton (Lamaze et al., 1997) and the later trafficking of endocytosed molecules is affected by disruption of the actin cytoskeleton (Durrbach et al., 1996). The mechanism by which actin is involved in endosomal trafficking is not well understood. There is also evidence to suggest that endomembrane trafficking and cell motility are somehow connected. Endocytosed molecules are preferentially exocytosed at sites of plasma membrane protrusions (Bretscher and Aguado-Velasco, 1998). Endosomes could transport molecules needed for cell motility to sites of active actin polymerization at the leading edge.

Cortactin is a widely expressed actin-binding protein that was originally identified as a substrate for src kinase (Wu et al., 1991). In addition to the actin-binding domain it contains an SH3-domain, several tyrosine phosphorylation sites and a proline-rich region (Wu et al., 1991). It interacts via its SH3domain with several PDZ-family proteins (Du et al., 1998; Katsube et al., 1998; Naisbitt et al., 1999). Cortactin has been implicated in cell motility and in linking transmembrane signaling to the cytoskeleton (Huang et al., 1998; Kinnunen et 
al., 1998). It has an F-actin cross-linking activity that is regulated by src kinase-mediated phosphorylation (Huang et al., 1997).

To further understand the function of cortactin, we examined its dynamics in live cells by expressing fluorescent protein tagged versions of this protein. In addition, its relationship to actin and arp2/3 complex was studied. We present evidence that shows the association of cortactin with dynamic actin at plasma membrane protrusions and on endosomal vesicles. These results suggest a role for cortactin as a linker of transmembrane receptors and actin cytoskeleton at the plasma membrane and on endosomes.

\section{MATERIALS AND METHODS}

\section{DNA constructs and transfection}

Mouse cortactin cDNA was amplified by PCR and cloned into pEGFPN1, pEGFP-C1 or pDsRed1-N1 (for red fluorescent protein, RFP) vector (Clontech, Palo Alto, CA) and verified by sequencing. Mouse cDNA used as a template was a kind gift from T. Parsons. NIH 3T3 cells were transfected using Fugene 6 transfection reagent according to manufacturer's recommendations (Boehringer Mannheim, Indianapolis, IN). Transiently transfected cells were used for experiments 24-48 hours after transfection. In addition, a stably transfected line obtained by G418 selection was used. GFP-arp3 and GFP-actin plasmids were kind gifts from D. Schafer (Schafer et al., 1998).

\section{Immunocytochemistry}

Cells were seeded on glass coverslips, allowed to attach and spread and then fixed with $2 \%$ paraformaldehyde in PBS. They were permeabilized with $0.1 \%$ Triton X-100 and stained with monoclonal antibody 4F11 (Upstate Biotechnology, Lake Placid, NY) for cortactin. Oregon-green-phalloidin or TRITC-phalloidin (Molecular Probes, Eugene, OR) was used to label F-actin.

\section{Microscopy of live cells}

Cells were seeded on glass coverslips in DMEM (without phenol red) containing $10 \%$ fetal calf serum and allowed to attach for 3-4 hours. The coverslips were then sealed with the same medium on a microscope slide with vaseline and small coverslip pieces as spacers. Olympus Provis microscope equipped with $\times 100$, NA 1.3 or $\times 60$, NA 1.4 objective and Sensys cooled CCD-camera (Photometrics, Tucson, AZ) were used for imaging. Fluorescent illumination was attenuated by neutral density filters to avoid phototoxicity. Thin areas of well spread cells were chosen for imaging in order to avoid out of focus haze. Imaging was done at room temperature. For endosomal labeling cells attached on coverslip were washed with serum-free medium, loaded with $10 \mu \mathrm{g} / \mathrm{ml}$ TRITC-transferrin (Molecular Probes) in serum free medium for 1 hour at $+37^{\circ} \mathrm{C}$, washed extensively with growth medium and immediately processed for imaging. Image Pro Plus (Media Cybernetics, Silver Spring, MD) program was used for image acquisition and images were further processed with Adobe Photoshop (Adobe Systems, San Jose, CA).

\section{Online supplemental material}

The online version of this article includes videos that correspond to Figs 2 (video 1), 3 (video 2) and 5 (video 3). Total acquisition time was 5 minutes for video 1 and 4 minutes for videos 2 and 3 . Videos are in the QuickTime format and are available at http://www.biologists.com/JCS/movies/jcs1893.html

\section{RESULTS}

\section{Localization of cortactin fusion proteins}

To study the localization and dynamics of cortactin in living cells we produced expression vectors of cortactin fused to GFP or RFP. We made vectors wherein the fluorescent protein was fused to either the $\mathrm{C}$ - or N-terminus of cortactin (pCortactinGFP, pCortactin-RFP and pGFP-Cortactin respectively). These constructs were then expressed in NIH $3 \mathrm{~T} 3$ fibroblasts, either in transiently transfected cells or in a stably expressing cell line. The localization of all three fusion proteins was identical to that of the endogenous cortactin as revealed by immunostaining (Fig. 1). Therefore, the fluorescent protein tag did not interfere with the normal localization of cortactin. Cortactin was localized, as shown in previous studies ( $\mathrm{Wu}$ et al., 1991), to the lamellipodia in spreading cells. It was also concentrated into small spots that were found throughout the cytoplasm. The localization of cortactin fusion proteins was clearly different from GFP alone, which was diffusely distributed (Fig. 1e,f). Co-expression of cortactin-RFP and soluble GFP in the same cells showed that the lamellipodial cortactin signal was not due to increased thickness at the leading edge but manifested true concentration of the cortactin fusion protein. Expression of the fusion proteins did not have any apparent effects on cell morphology.
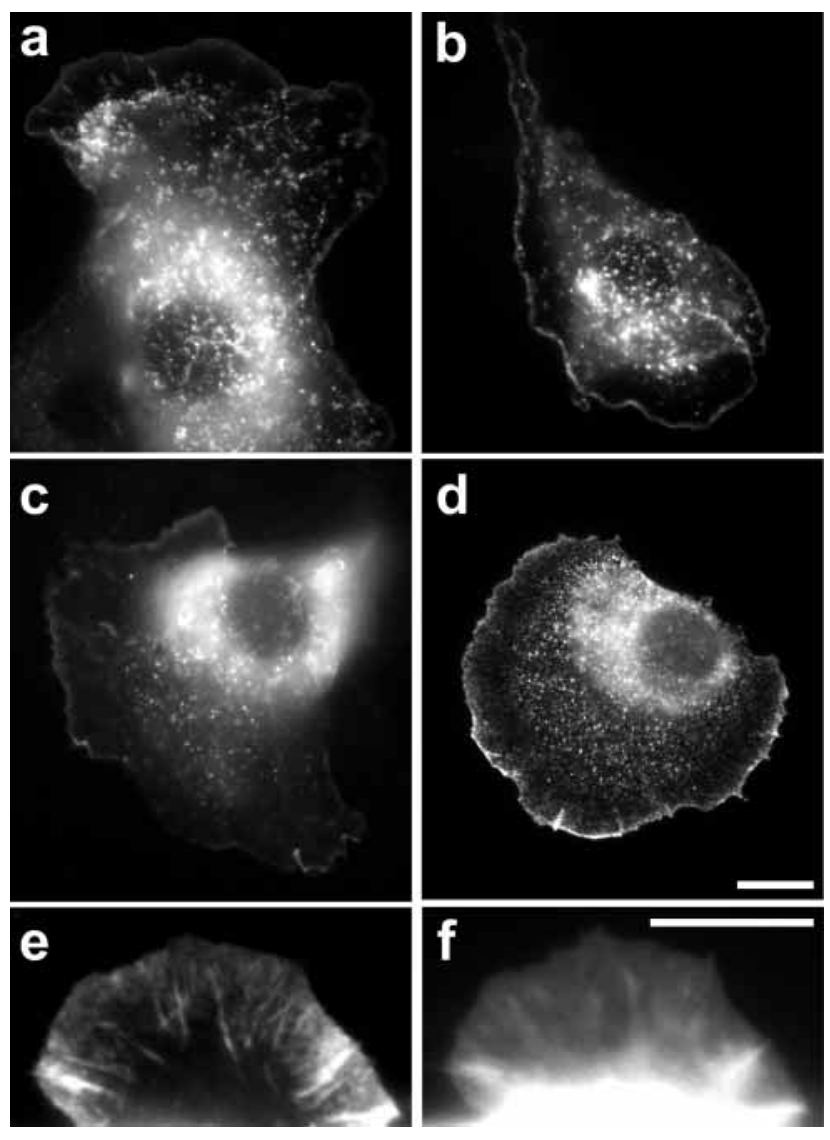

Fig. 1. Localization of GFP or RFP tagged cortactin is similar to endogenous cortactin. (a-c) Microphotographs of live NIH 3T3 cells expressing cortactin-GFP (a), cortactin-RFP (b) or GFP-cortactin (c) fusion protein. (d) NIH 3T3 cells fixed, permeabilized and immunostained for cortactin. (e-f) A live cell co-transfected with cortactin-RFP (e) and wild-type GFP (f). Unlike GFP, which exhibited a diffuse cytoplasmic fluorescence, cortactin-RFP was localized at filamentous structures at the leading edge. Bars, $10 \mu \mathrm{m}$. 


\section{Dynamics of cortactin fusion proteins}

Time-lapse analysis of cells expressing cortactin fusion proteins revealed that cortactin-labeled structures behaved in a strikingly dynamic manner. In the lamellipodia, cortactin was associated with structures that moved from the leading edge toward the cell center (Fig. 2, video 1). The speed of the movement, measured by tracking features brighter than the average lamellipodia staining, was $2.5 \mu \mathrm{m} /$ minute (s.d. \pm 0.33 , measured from 10 different cells). The directionality and speed of this movement are consistent with the reported retrograde-flow of F-actin meshwork in the lamellipodium of fibroblasts (Fisher et al., 1988; Theriot and Mitchison, 1992; Wang, 1985). Cortactin is thus likely to be associated with the F-actin meshwork in the lamellipodia. Cytoplasmic spots were also very dynamic. They, however, moved in a random manner and did not show any consistent direction. The spots were most abundant in the perinuclear area and their frequency of occurrence decreased toward the thin, lamellar parts (Figs 1, 2).

\section{Cortactin colocalizes with dynamic actin}

To further study the localization and dynamics of cortactin in comparison to actin we cotransfected cells with expression vectors encoding for cortactin-RFP and GFP-actin fusion proteins. GFP-actin distribution in the lamellipodia was less punctate than that of cortactin-RFP (Fig. 3). The retrograde movement of structures in the lamellipodia was seen with both fusion proteins. The area exhibiting retrograde flow was the same for both fusion proteins and so was the speed of the movement (about $3 \mu \mathrm{m} /$ minute).

Cytoplasmic spots stained by cortactinRFP were also weakly stained by GFP-actin. This was most clear in thin areas of the cells, while in the thicker parts, possible localization of GFP-actin to the cytoplasmic spots was masked by high concentration of the soluble form of GFP-actin and by brightly labeled F-actin bundles. Actin structures such as stress fibers that remained stable during time-lapse recording (4 minutes in duration) were not stained by cortactin-RFP (Fig. 3, video 2). Cortactin is thus associated only with a sub-set of F-actin that is very dynamic. Colocalization of cortactin and F-actin was confirmed by phalloidin staining of fixed cortactin-GFP expressing cells (Fig. 4a-c). As phalloidin binds only to filamentous actin the staining shows that cortactin spots contain F-actin.

\section{Cortactin colocalizes with arp2/3 complex}

Arp2/3 complex has a crucial role in the regulation of actin polymerization and has been shown to localize to sites of dynamic actin (Machesky and Gould, 1999; Schafer et al., 1998). We therefore studied whether cortactin co-localized with the arp2/3 complex. Cells were co-transfected with vectors encoding GFP-arp3 and cortactin-RFP fusion proteins. Imaging of these cells revealed that the two proteins were co-localized at the lamellipodia and at the cytoplasmic spots (Fig. 4d-f). Cortactin-RFP fusion protein, however, gave a much stronger signal than GFP-arp3. This may be due to a higher number of binding sites for cortactin or because the GFP-arp3 fusion protein is not efficiently incorporated into the arp2/3 complex. The movement of cortactin-GFP labeled structures stopped when actin polymerization was inhibited by cytochalasin D (data not shown) as was also shown for GFP-arp3 labeled structures (Schafer et al., 1998). Thus, the cortactin fusion protein can be used as a marker for dynamic F-actin.

\section{Cortactin associates with endosomes}

The cytoplasmic cortactin spots were seen in some cells to be associated with vesicular structures that were visible as dark spheres against the diffuse cytoplasmic fluorescence due to the soluble pool of the fusion protein (Fig. 5a-i). The vesicles were most clearly seen in cells that expressed high levels of cortactin fusion protein and therefore had an intense cytoplasmic

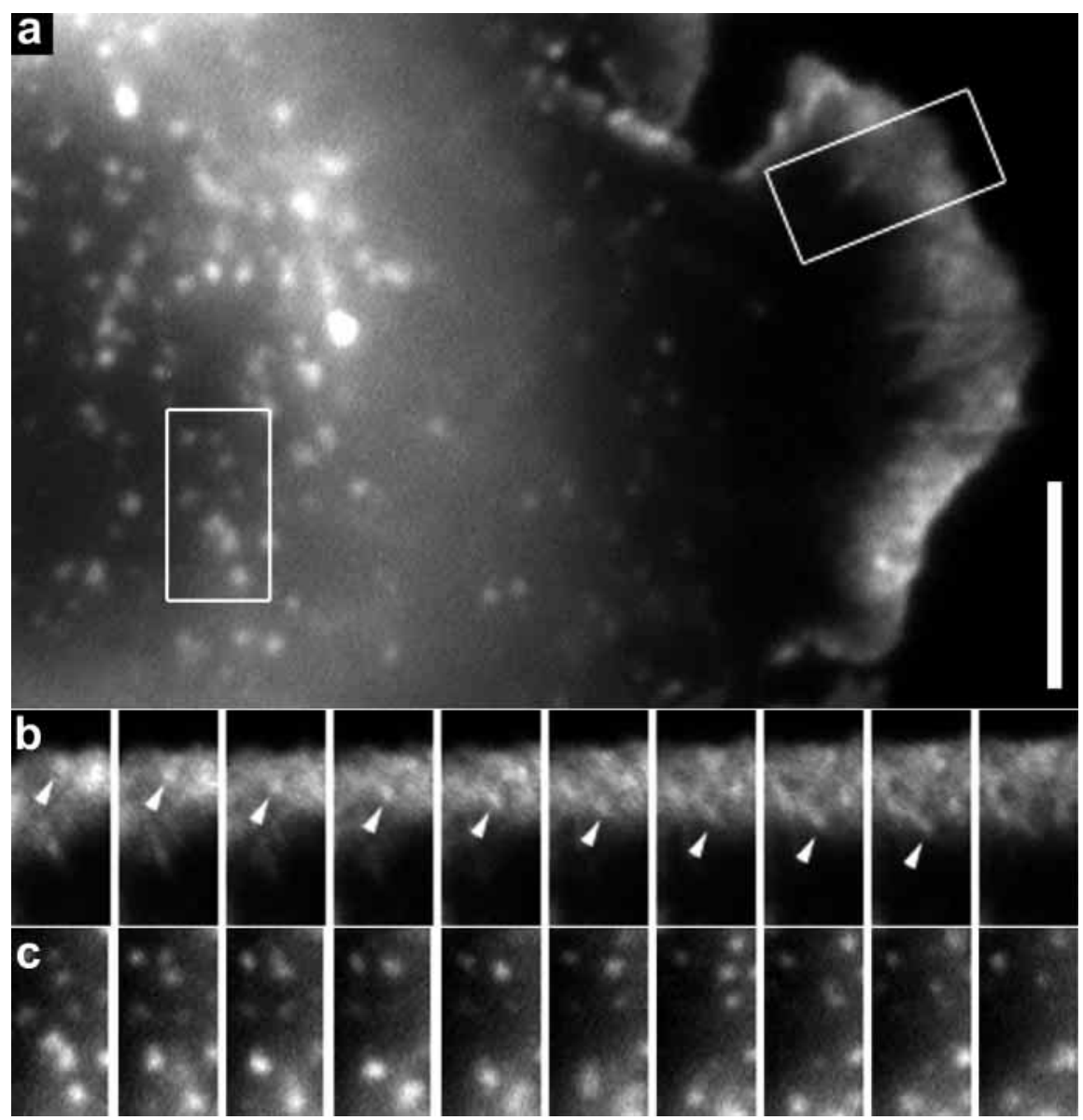

Fig. 2. Dynamic behavior of cortactin labeled structures. (a) a general view of a cortactinRFP expressing cell. Cell center is on the left and a cortactin-RFP labeled lamellipodium is on the right. (b) Time-lapse series reveals the retrograde movement of cortactin in the lamellipodium (boxed area on the right in a). Arrowhead points to a bright spot that moves from the leading edge toward the cell center. (c) Time-lapse series showing the random movements of the cytoplasmic spots (boxed area on the left in a). Images were taken at intervals of 6 seconds. Bar, $5 \mu \mathrm{m}$. 

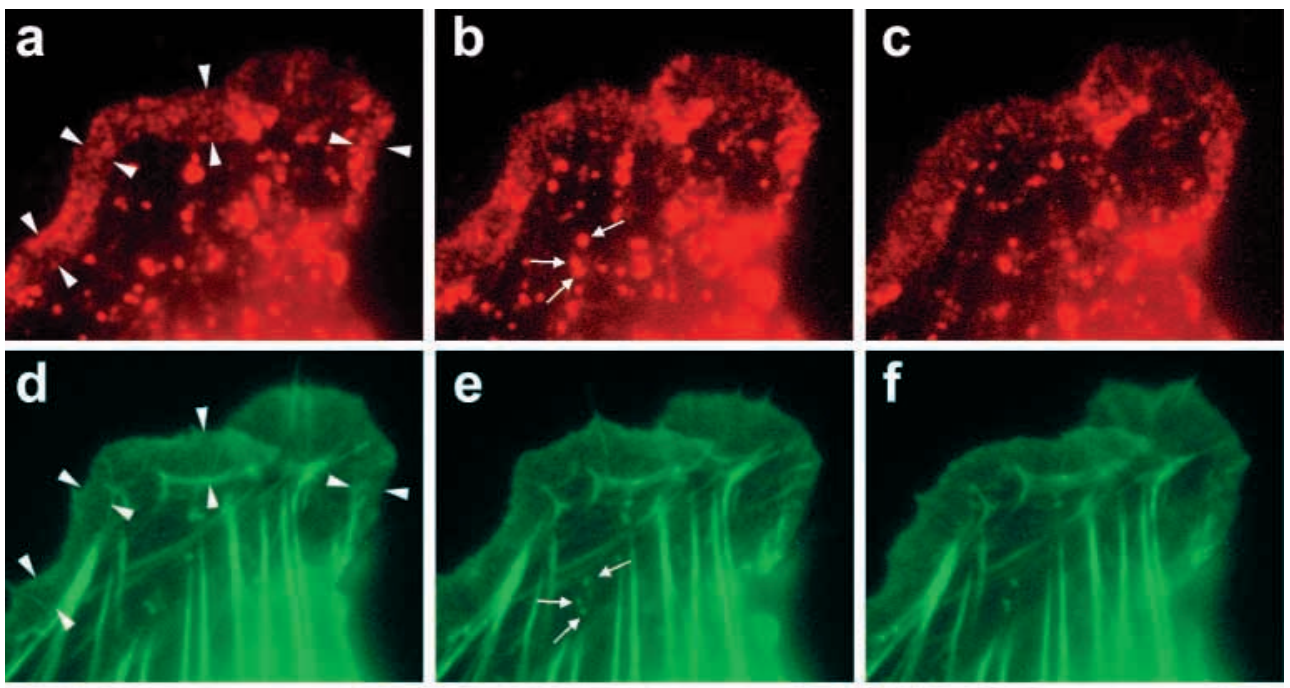

Fig. 3. Visualization of cortactin-RFP and GFP-actin in living cells. A cell expressing cortactin-RFP (a-c) and GFP-actin (d-f) was imaged for 4 minutes. Images from the beginning, middle and end of the series are shown here. Arrowheads (a,d and g) indicate the area where retrograde movements are seen. Arrows (b, e and $\mathrm{h}$ ) show cytoplasmic spots that are labeled both by cortactin-RFP and GFP-actin. Bar, $10 \mu \mathrm{m}$.
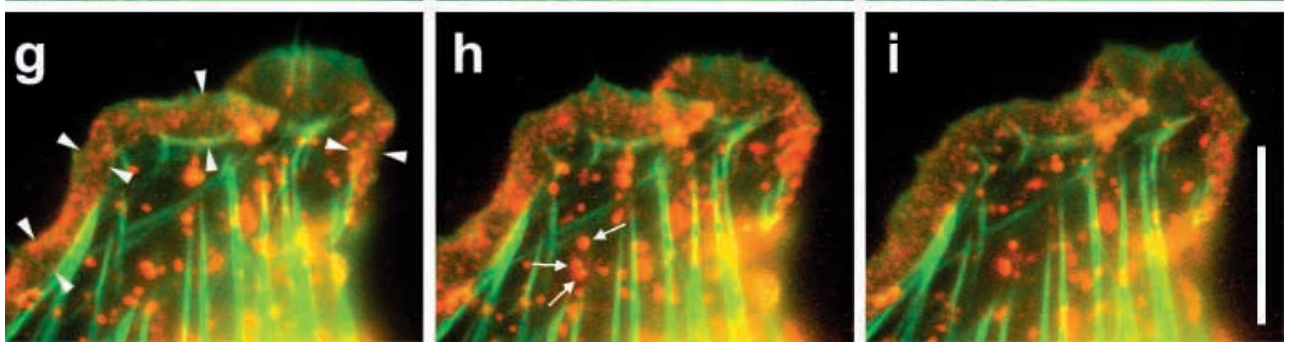

staining. The vesicles were not completely covered by cortactin-GFP but were asymmetrically associated with one or a few cortactin-GFP spots. When these vesicles moved, they were associated with short comet tails of cortactin-GFP at the trailing edge of these vesicles. Fig. 5a-i (see also video 3) shows a representative time-lapse series. The upper vesicle (arrowhead in 5a) moved initially downward while its cortactin-GFP tail pointed upward. Then the vesicle turned 180 degrees and the cortactin spot now pointed downward (5d). This was followed by an upward movement of the vesicle (5di). Two lower vesicles (arrows in 5a) moved slowly toward each other until they fused into one. Fusion was accompanied by an increase in the vesicular diameter. The two cortactin spots associated with the fused vesicle coalesced shortly after the fusion event (5e). The cortactin spot then turned downward and the vesicle started moving upward (5e-i). Cortactin spots were, however, not seen to be associated with sites of vesicle fusion.

To characterize the identity of the vesicles associated with cortactin, we loaded the cells with markers of endocytosis. Cortactin-GFP-expressing cells were incubated with fluorescently labeled transferrin. Transferrin binds to cell surface transferrin receptors and is endocytosed through receptor-mediated endocytosis via coated pits. It is then recycled back to the plasma membrane via recycling endosomes (Robinson et al., 1996). Imaging transferrin and cortactin-GFP together clearly revealed that endosomal vesicles were associated with cortactin-GFP spots (Fig. 5j). Over half of transferrin-labelled vesicles were associated with cortactin-GFP tails $(59.0 \%$, s.e.m. $\pm 4.3 \%$, calculated from randomly chosen microscopic fields from 10 different cells). The majority of vesicles associated with cortactin-GFP had only one asymmetric cortactin-GFP tail (74.3\%, s.e.m. $\pm 1.9 \%)$
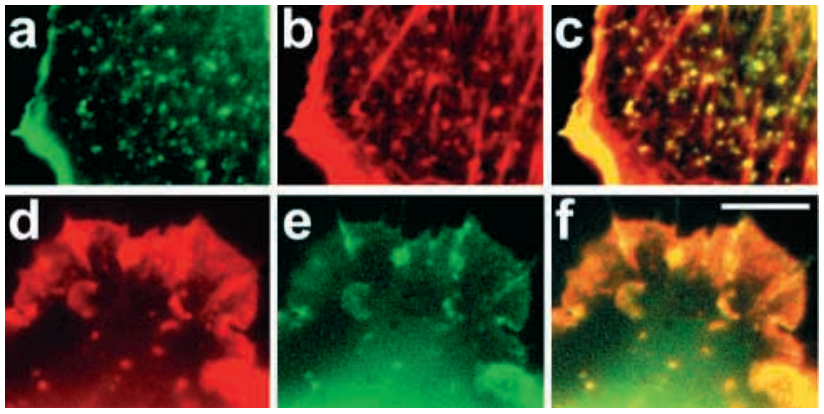

Fig. 4. Cortactin-GFP labeled structures contain F-actin and arp3. (a-b) Localization of cortactin-GFP (green) and F-actin stained by TRITC-phalloidin (red) in a fixed and permeabilized cell.

(c) Superimposition of images a and b shows colocalization of cortactin-GFP with F-actin in lamellipodium and at the cytoplasmic spots. (d-e) Localization of cortactin-RFP (red) and GFP-arp3

(green) in a living cell. (f) Superimposition of images $d$ and e reveals colocalization at the lamellipodium and at the cytoplasmic spots. Bar, $5 \mu \mathrm{m}$.

and fewer had two or more tails $(25.7 \pm 1.9 \%)$. No vesicles were found to be totally covered by cortactin-GFP. Similar results were obtained when the cells were loaded with fluorescent BSA, which functions as a marker for fluid-phase endosytosis (Fig. 5k).

F-actin tails were also seen when non-transfected cells were loaded with fluorescent transferrin, fixed and stained with fluorescent phalloidin (Fig. 51). This excludes the possibility that the tails were artifacts caused by cortactin-GFP expression. Imaging of endosomal F-actin tails in live cells using GFPactin expression was hampered by the high cytoplasmic 
Fig. 5. Asymmetric association of endosomal vesicles with cortactin spots.

(a-i) Movement of vesicles with cortactin-GFP tails. The total length of the time series is 4 minutes and interval between each image is 30 seconds. See text for detailed discussion. Bar, $2.5 \mu \mathrm{m}$. (j) Cortactin-GFP (green) and TRITC-transferrinlabeled endosomes (red).

(k) Cortactin-GFP (green) and Texas Red-BSA-labeled
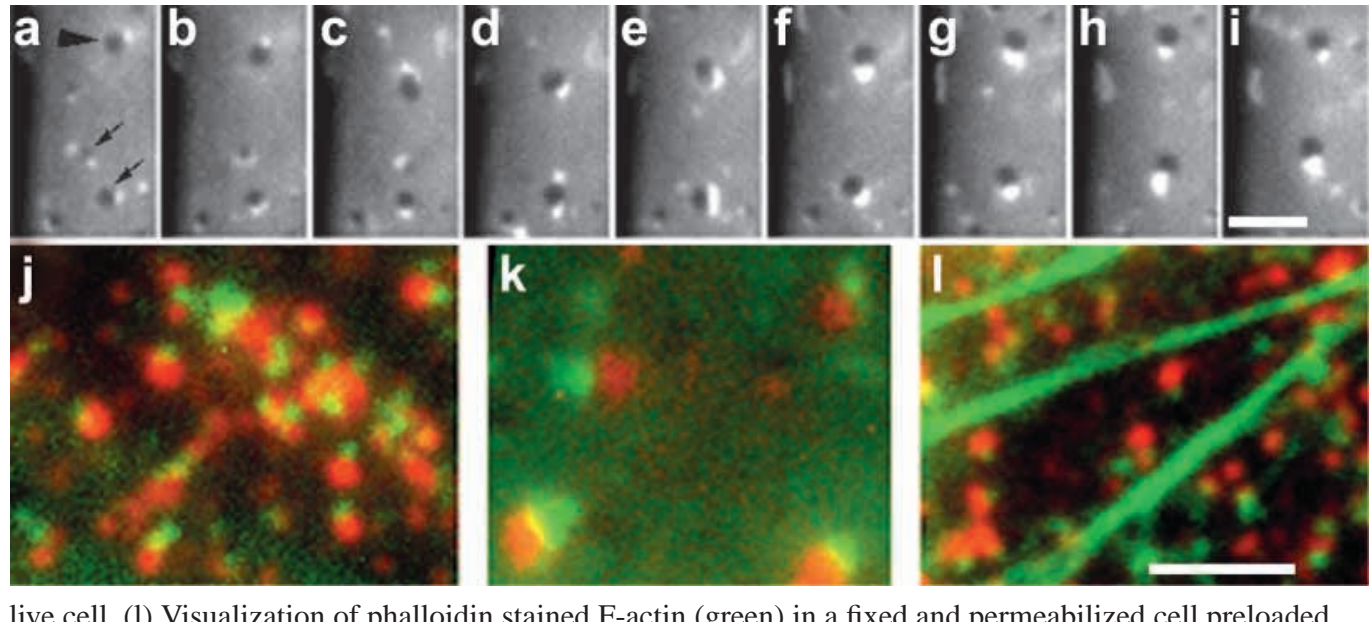

endosomes (red) as visualized in a live cell. (1) Visualization of phalloidin stained F-actin (green) in a fixed and permeabilized cell preloaded with TRITC-transferrin to stain endosomes (red). Bar, $2.5 \mu \mathrm{m}$.

concentration of diffuse G-actin and by other F-actin structures such as stress fibers.

\section{DISCUSSION}

We show in this study that cortactin localizes to two different dynamic membrane structures, lamellipodia and endosomes. Cortactin is associated in these structures with F-actin that undergoes rapid turnover. Both of these membrane domains are propelled by actin polymerization.

Myosin-driven retrograde flow of actin meshwork in the lamellipodium is a crucial mechanism for cell migration (Lin et al., 1996). Linking of the actin meshwork to the underlying extracellular matrix via transmembrane receptors is thought to result in the forward movement of the cell (Lin and Forscher, 1995; Suter et al., 1998). We have shown that cortactin is associated with the F-actin meshwork in the lamellipodium. As cortactin interacts with both F-actin and transmembrane receptors it may be involved in linking these two systems together. Cortactin binds to polyproline motifs in several different PDZ domain proteins via its SH3 domain (Du et al., 1998; Katsube et al., 1998; Naisbitt et al., 1999). PDZ proteins assemble signaling complexes at the plasma membrane, and they interact specifically with a wide variety of transmembrane proteins using their PDZ domains (Fanning and Anderson, 1999). Cortactin may function in lamellipodia as a linker of actin meshwork and transmembrane receptors via PDZproteins and thus connect the actin meshwork to the underlying substrate. Src kinases are known to regulate the F-actin crosslinking activity of cortactin (Huang et al., 1997) and overexpression of a cortactin mutant deficient in tyrosine phosphorylation impaired the migration of endothelial cells (Huang et al., 1998). Cortactin could thus provide a mechanism for linking src signaling with cell motility.

The actin cytoskeleton is essential at several steps in the endocytic cycle. However, the precise role of actin in endosomal traffic has been unclear. Our finding that endosomal vesicles are asymmetrically associated with and move with dynamic actin patches suggests that the propulsive force derived from actin polymerization is used for trafficking endocytic vesicles in a manner similar to its involvement in propelling plasma membrane protrusions and intracellular pathogens. Asymmetric association of F-actin patches around the vesicles, which is obviously necessary for movement, may be a spontaneous property of dynamic actin network as suggested by recent experiments using cell extracts and polystyrene beads coated with bacterial protein that induces actin polymerization (van Oudenaarden and Theriot, 1999). Factin is required for the initial formation of endocytic vesicles at the plasma membrane (Lamaze et al., 1997; Durrbach et al., 1996). Recently it has been shown, using GFP-actin expression, that macropinosomes generated at plasma membrane ruffles of cultured mast cells, are transiently associated with actin tails when they move from the plasma membrane towards the cytoplasm (Merrifield et al., 1999). Actin cytoskeleton is, however, also required for later cytoplasmic trafficking of endosomes (Durrbach et al., 1996). Actin rocketing of endosomes was also very recently shown in Xenopus oocytes (Taunton et al., 2000) and in cultured mammalian cells activated by overexpression of phosphatidylinositol phosphate 5-kinase or by pervanadate application (Rozelle et al., 2000). Actin comet tails described in these studies were more robust but less frequently observed than those shown here. Our results suggest that actin rocketing of endosomes is, in fact, a common mechanism for endosome trafficking.

Transmembrane receptors, such as epidermal growth factor receptor (EGFR), may also be involved in endosomal trafficking. Activated EGFR can induce actin polymerization at the plasma membrane (Rijken et al., 1991). The activated receptor is endocytosed and its activity is known to persist in endosomes (Haugh et al., 1999). It is thus possible that it could also induce actin polymerization at endosomal membranes. Further, cortactin could link transmembrane signaling at endosomal membranes to $\mathrm{F}$-actin in a manner proposed to occur at the plasma membrane.

As endosomes and the leading edge contain similar molecular components that are required for actin polymerization, it is concievable that localized exocytosis of recycled endosomes may regulate direction of actin based cell motility by concentrating membrane associated molecules that induce actin polymerization. In migrating cells the leading edge is known to be the major site of exocytosis and an essential role 
for membrane recycling in cell motility has recently been suggested (Bretscher and Aguado-Velasco, 1998).

We thank Dr Dorothy Shaffer for the gifts of GFP-arp3 and GFPactin plasmids and Dr Tom Parsons for mouse cortactin cDNA. We thank Dr E. Kuismanen, and members of the Peng lab for comments and discussion. We also thank Eeva-Liisa Saarikalle for assistance in preparation of DNA constructs. This work was supported by grants from NIH, MDA (H.B.P.), the Juselius Foundation (M.K., H.B.P. and H.R.) and The Finnish Academy (Programme of molecular neuroscience) (M.K. and H.R.).

\section{REFERENCES}

Bretscher, M. S. and Aguado-Velasco, C. (1998). Membrane traffic during cell locomotion. Curr. Opin. Cell Biol. 10, 537-541.

Du, Y., Weed, S. A., Xiong, W. C., Marshall, T. D. and Parsons, J. T. (1998). Identification of a novel cortactin SH3 domain-binding protein and its localization to growth cones of cultured neurons. Mol. Cell Biol. 18, 58385851.

Durrbach, A., Louvard, D. and Coudrier, E. (1996). Actin filaments facilitate two steps of endocytosis. J. Cell Sci. 109, 457-465.

Fanning, A. S. and Anderson, J. M. (1999). Protein modules as organizers of membrane structure. Curr. Opin. Cell Biol. 11, 432-439.

Fisher, G. W., Conrad, P. A., DeBiasio, R. L. and Taylor, D. L. (1988). Centripetal transport of cytoplasm, actin, and the cell surface in lamellipodia of fibroblasts. Cell Motil. Cytoskel. 11, 235-247.

Haugh, J. M., Huang, A. C., Wiley, H. S., Wells, A. and Lauffenburger, D. A. (1999). Internalized epidermal growth factor receptors participate in the activation of p21(ras) in fibroblasts. J. Biol. Chem. 274, 34350-34360.

Huang, C., Ni, Y., Wang, T., Gao, Y., Haudenschild, C. C. and Zhan, X. (1997). Down-regulation of the filamentous actin cross-linking activity of cortactin by Src-mediated tyrosine phosphorylation. J. Biol. Chem. 272, 13911-13915.

Huang, C., Liu, J., Haudenschild, C. C. and Zhan, X. (1998). The role of tyrosine phosphorylation of cortactin in the locomotion of endothelial cells. J. Biol. Chem. 273, 25770-25776.

Katsube, T., Takahisa, M., Ueda, R., Hashimoto, N., Kobayashi, M. and Togashi, S. (1998). Cortactin associates with the cell-cell junction protein ZO-1 in both Drosophila and mouse. J. Biol. Chem. 273, 29672-29677.

Kinnunen, T., Kaksonen, M., Saarinen, J., Kalkkinen, N., Peng, H. B. and Rauvala, H. (1998). Cortactin-Src kinase signaling pathway is involved in N-syndecan-dependent neurite outgrowth. J. Biol. Chem. 273, 10702-10708.

Lamaze, C., Fujimoto, L. M., Yin, H. L. and Schmid, S. L. (1997). The actin cytoskeleton is required for receptor-mediated endocytosis in mammalian cells. J. Biol. Chem. 272, 20332-20335.
Lin, C. H. and Forscher, P. (1995). Growth cone advance is inversely proportional to retrograde F-actin flow. Neuron 14, 763-771.

Lin, C. H., Espreafico, E. M., Mooseker, M. S. and Forscher, P. (1996) Myosin drives retrograde F-actin flow in neuronal growth cones. Neuron 16 769-782.

Machesky, L. M. and Gould, K. L. (1999). The Arp2/3 complex: a multifunctional actin organizer. Curr. Opin. Cell Biol. 11, 117-121.

Merrifield, C. J., Moss, S. E., Ballestrem, C., Imhof, B. A., Giese, G. Wunderlich, I. and Almers, W. (1999). Endocytic vesicles move at the tips of actin tails in cultured mast cells. Nature Cell Biol. 1, 72-74.

Mitchison, T. J. and Cramer, L. P. (1996). Actin-based cell motility and cell locomotion. Cell 84, 371-379.

Naisbitt, S., Kim, E., Tu, J. C., Xiao, B., Sala, C., Valtschanoff, J., Weinberg, R. J., Worley, P. F. and Sheng, M. (1999). Shank, a novel family of postsynaptic density proteins that binds to the NMDA receptor/PSD-95/GKAP complex and cortactin. Neuron 23, 569-582.

Rijken, P. J., Hage, W. J., van Bergen en Henegouwen P. M., Verkleij, A. J. and Boonstra, J. (1991). Epidermal growth factor induces rapid reorganization of the actin microfilament system in human A431 cells. $J$. Cell Sci. 100, 491-499.

Robinson, M. S., Watts, C. and Zerial, M. (1996). Membrane dynamics in endocytosis. Cell 84, 13-21.

Rozelle, A. L., Machesky, L. M., Yamamoto, M., Driessens, M. H., Insall, R. H., Roth, M. G., Luby-Phelps, K., Marriott, G., Hall, A. and Yin, H. L. (2000). Phosphatidylinositol 4, 5-bisphosphate induces actin-based movement of raft-enriched vesicles through WASP-Arp2/3. Curr. Biol. 10, 311-320.

Schafer, D. A., Welch, M. D., Machesky, L. M., Bridgman, P. C., Meyer, S. M. and Cooper, J. A. (1998). Visualization and molecular analysis of actin assembly in living cells. J. Cell Biol. 143, 1919-1930.

Suter, D. M., Errante, L. D., Belotserkovsky, V. and Forscher, P. (1998). The Ig superfamily cell adhesion molecule, apCAM, mediates growth cone steering by substrate-cytoskeletal coupling. J. Cell Biol. 141, 227-240.

Taunton, J., Rowning, B. A., Coughlin, M. L., Wu, M., Moon, R. T., Mitchison, T. J. and Larabell, C. A. (2000). Actin-dependent propulsion of endosomes and lysosomes by recruitment of N-WASP. J. Cell Biol. 148 519-530.

Theriot, J. A. and Mitchison, T. J. (1991). Actin microfilament dynamics in locomoting cells. Nature 352, 126-131.

Theriot, J. A. and Mitchison, T. J. (1992). Comparison of actin and cell surface dynamics in motile fibroblasts. J. Cell Biol. 119, 367-377.

van Oudenaarden, A. and Theriot, J. A. (1999). Cooperative symmetrybreaking by actin polymerization in a model for cell motility. Nature Cell Biol. 1, 493-499.

Wang, Y. L. (1985). Exchange of actin subunits at the leading edge of living fibroblasts: possible role of treadmilling. J. Cell Biol. 101, 597-602.

Wu, H., Reynolds, A. B., Kanner, S. B., Vines, R. R. and Parsons, J. T. (1991). Identification and characterization of a novel cytoskeletonassociated pp60src substrate. Mol. Cell Biol. 11, 5113-5124. 